\title{
An Enhancement of Underwater Images using DCP and CLAHE Algorithm
}

\author{
Malathi V, Manikandan A
}

\begin{abstract}
The lack of resource requirement in this population world, we are in a position to require another resources. In this regard, ocean is one of our sustenance. It is the exact platform for various applications like, transport, food, energy etc., but still we are surveyed partly at all aspects. One of the main focus of challenge is scattering of light as it penetrate from air to water which presents us with a bluish background while studying the scenery. In this, added to this there is a hazy appearance in the visuals and calls for Image Enhancement techniques. Here, Dark Channel Prior(DCP) is used to remove the haze and noise induced by the bluish environment. However, this proposal of method is also used to increase darkness of the image, Contrast Limited Adaptive Histogram Equalization (CLAHE) is used on the RGB image to enhance the contrast and intensity of the image. Finally, we get visually pleasing result, colour correlation method is carried out. The experimental result shows that a enhanced underwater image from the base image, and mostly useful to analyze and monitoring the underwater images.
\end{abstract}

Index Terms-CLAHE, Dark channel prior, Haze, Image Enhancement, RGB

\section{INTRODUCTION}

In order to maintain a balance between the usage of underwater resources and the ecosystem continuous surveillance of the underwater scenario is being done. Here the underwater imaging finds a large scale application. Unfortunately, under- water images are loss of colour, poor contrast, presence of a bluish and a hazy background. Refraction and absorption by water that it's responsible for changes in colour and bluish tone in the image. Each colour absorbs at a different rate because it has a different wavelength and energy level. The factor of depth also plays a role here. A deeper and deeper under the sea the colours with larger wavelengths are absorbed more. Colours are absorbed more than blue and green causing a bluish tone to the image. In general, underwater images generally suffer from poor visibility due to the attenuation of the propagated light. The haphazard attenuation of the light underwater causes a foggy and misty appearance of the distant object in the image and the scene is characterized by poor contrast. To improve the quality of such images and scenes, image enhancement needs to be done.

Revised Manuscript Received on December 30, 2019.

* Correspondence Author

Malathi V, Assistant Professor, SRM Arts And Science And College, Chennai, Tamil Nadu, India.

E-mail Malathikb@Gmail.Com

Dr. Manikandan A, Assistant Professor, Muthayammal Memorial College Of Arts And Science, Rasipuram, Tamil Nadu, India. E-mail S.A.Manikandan@Gmail.Com

(C) The Authors. Published by Blue Eyes Intelligence Engineering and Sciences Publication (BEIESP). This is an open access article under the CC BY-NC-ND license (http://creativecommons.org/licenses/by-nc-nd/4.0/)
Image enhancement is a technique used to enhance the optical quality of images to ensure better visual quality/ extraction of information from the image. [1] suggests a method to enhance underwater videos and images using fusion principles. [2] presented a new technique to estimate the optical transmission in hazy images. Using this estimation, the scattered light is eliminated to increase scene visibility and recover haze-free scene contrasts. [3] Proposed a new method called mixture contrast limited adaptive histogram equalization (Mix- CLAHE) colour models where the method is operated on both RGB and HSV colour models. However, this algorithm is over amplifies the objects and noise in the final image. Image dehazing is a process to remove haze or fog effects from the poor quality images [4]. It is an image enhancement technique which is usually used to remove haze. [5] proposed a simple but effective image dehazing algorithm; Dark Channel Prior(DCP) algorithm is used to remove haze from a single input image. But, the defect in this algorithm is that tends to darken the image and reduces contrast [6]. Here, we proposed a new algorithm, that is integration of DCP and CLAHE with further colour correlation technique to produce a more visually pleasing image.

This paper is divided into six sections with section I being the Introduction. In section II, we take a look at the existing methods, Section III focuses on the detailed view of CLAHE and DCP, Section IV deals with Proposed Algorithm, section $\mathrm{V}$ involves Simulation and performance evaluation. Finally in Section VI, we conclude the proposed algorithm performance from the experimental result.

\section{IMAGE ENHANCEMENT METHODS}

\section{A. Homomorphic Filtering}

This method is used to fix the non-uniform lighting in an image which needs its contrast to be reinforced [7]. It is a frequency filtering technique

$F(x, y)=I(x, y) * r(x, y)$

where $F(x, y)$ is the function of the image detected, $I(x, y)$ is the illumination function and $r(x, y)$ is the reflectance. Here the image is Fourier transformed, multiplied with a filter function and then transformed back into the spatial domain. By multiplying these components filter can reduce nonuniform illumination. Mostly used this methods as it addresses the non- uniform illumination and sharpens the image. 


\section{An Enhancement of Underwater Images using DCP and CLAHE Algorithm}

\section{B. Anisotropic Filtering}

In this method the image components are disentangled to enhance image division. It is used to decrease compositions and diminishes the objects by erasing little edges enhanced by homomorphic filtering. This method also deals with low contrast and non-uniform illumination problem in images [3].

\section{Wavelet Denoising}

This method is used to reduce high frequency noise in images. A wavelet transform is computed from the original image. Noise level at each wavelet is estimated separately and a threshold is defined for zeroing wavelet co-efficient. Other co-efficients are shunted and an inverse wavelet transform is performed. Additionally, the Fourier transform tells about the frequency Components (Sine and Cosine) and their amplitudes, the wavelets also tell us where they exist in the time axis. It gives us a better Peak Signal to Noise Ratio (PSNR) and Mean Square Error (MSE) results compared to Homomorphic and Anisotropic Filtering.

\section{Red Channel Method}

Colours associated with Short wavelengths which were lost due to absorption are recovered leading to a recovery of lost contrast. It can be considered as a variant to the Dark Channel Prior Method. A Pixel that lies at a maximum depth with respect to the camera is first chosen. It is assumed that degradation of the image depends upon location of pixel. Transmission map of the scene is estimated and then colour correlation is made for visually pleasing results

\section{E. Histogram Equalization}

It is modifying the image intensities and contrast using the histogram of the image. When the obtained image of the underwater scenery has both the background and the frontal areas as both bright or both dark, this method is used to enhance the image. The key in this method is mapping the gray levels with the probability distribution of the available gray levels. One inconvenience in this method can be over amplification of the background $\mathrm{n}$ noise and decrease in the useful signal.

\section{III.DCP AND CLAHE ALGORITHM}

In this section we look into the DCP and CLAHE algorithms. First the image is subjected to a haze removal algorithm which is the DCP and further is enhanced by the CLAHE technique [8]

\section{A. Haze removal using Dark Channel Prior(DCP)}

This is used to obtain a natural Haze free image and is generally used in the process of image enhancement [9]. The haphazard attenuation of light by water particles as we go deeper and deeper into the sea causes a foggy and misty appearance to the image of the scenery. A hazy image can be characterized by using a function $\mathrm{S}(\mathrm{x})$ is given by

$$
S(x)=Z(x) t(x)+A(1-t(x))
$$

where $S(x)$ is the haze containing image, $Z(x)$ is the haze free image, $\mathrm{t}(\mathrm{x})$ is the transmission map and $\mathrm{A}$ is the global atmospheric light.
The ultimate focus of DCP is to find the haze image $\mathrm{Z}(\mathrm{x})$ from the haze containing image $S(x)$. There are 4 steps in the dehazing process [10] which includes

(i) estimation of atmospheric light

(ii) Transmission Map Estimation

(iii)Transmission Map Refinement and

(iv)image reconstruction.

[5]proposes that most of the local regions in the background of the image often have some pixels which have a very low intensity in one of the three channels of the (RGB). It can be denoted as ( $\mathrm{x}$ ) and it is considered as the dark channel at $\mathrm{x} \mathrm{x}$ can be denoted as

$$
Z^{\text {dark }}(x)=\min _{c e R G B} \min _{y \propto \Omega(x)} Z^{C}(y)
$$

where $Z^{c}$ is one of the channels in $\mathrm{Z}$ and (x) is a square region with center $\mathrm{x}$. If the square region does not belong to any local regions, then tends to zero. Hence, it is called dark channel. $\mathrm{S}(\mathrm{x})$ is an image whose intensity mixed with atmospheric light. So it is usually brighter than $\mathrm{Z}(\mathrm{x})$. So the dark channel of $S(x)$ is brighter than the dark channel of $\mathrm{Z}(\mathrm{x})$. This fact serves as a useful result in the estimation of $\mathrm{Z}(\mathrm{x})$.Substituting (3) in (2) we get

$$
\min _{c e R G B} \min _{y \delta \Omega(x)} s^{c}(y)=\min _{c e R G B} \min _{y \varepsilon \Omega(x)} z^{c}(y) t(x)+A^{C}(1-t(x))
$$

follow the four steps of estimation in DCP. For computing the value of $\mathrm{A}$, the highest $0.1 \%$ of brightest pixels in the dark channel is selected and the colour with the highest intensity value among the selected pixels is selected as the value of A. Now dividing both LHS and RHS of (4) by Ac, it gives

$$
\min _{c e R G B} \min _{y \sigma \Omega(x)} s^{C}(y) / A^{c}=\min _{c e R G B y \propto \Omega(x)} \min ^{C}(y) / A^{c} t(x)+(1-t(x))
$$

Now for Dark channel Prior the Dark Channel for the image without Haze tends to zero. i.e..

$$
\begin{aligned}
& \min _{c e R G B} \min _{y \curvearrowleft(x)} s^{c}(y) / A^{c} \\
& \text { Combining equation (5) and (6) we get, } \\
& t(x) 1-\min _{c e R G B} \min _{y \curvearrowleft(x)} S^{c}(y) / A^{c}
\end{aligned}
$$

[5] introduces a factor ' $w$ ' to keep a small amount of haze in the image to perceive the depth of the object. Hence $\mathrm{t}(\mathrm{x})$ can be computed as,

$$
\mathrm{t}(\mathrm{x})=1-\mathrm{w} \min _{\text {ceRGB }} \min _{y \boldsymbol{\alpha}(\mathrm{x})} S^{c}(y) / A^{c}
$$

This is the second step in dehazing using DCP. The next step is refining the transmission map $\mathrm{t}(\mathrm{x})$. Usually a Soft mapping is done to refine the transmission map. Finally the last step is the reconstruction of the haze free image. 


$$
Z(x)=\left[S(x)-A / \max \left(t(x), t_{0}\right)\right]+A
$$

Where $t_{0}$ is the threshold value applied to avoid low value of denominator

\section{A. Demerits of in Dark Channel Prior}

There are major defects occurred in DCP. Initially, it will be consider from the background. In any kind of image, object and the background. After DCP, where there is a removal of haze, there is very low contrast with the background and sometimes this may also darken the local regions of the image. This is because background may also be mixed with the haze. The $\mathrm{Z}(\mathrm{x}) \mathrm{t}(\mathrm{x})$ is called direct attenuation and has a non-linearity with the intensity of the image. After DCP is performed, the resultant image has a lower contrast than $\mathrm{Z}(\mathrm{x})$. [1-t(x)] represents the thickness of the haze present in the image. For different values of $\mathrm{A}$ under the same attenuation and thickness of haze also results in an image which is lower in contrast of the Haze free image $\mathrm{Z}(\mathrm{x})$. These are overcome by performing the CLAHE algorithm.

Contrast Limited Adaptive Histogram Equalization (CLAHE):

This method is also used to enhance the contrast of images. This is a derivation of the Adaptive histogram equalization (AHE). Here, the contrast of the image has constant and histogram equalization. The image is divided into tiles. Tiles are small regions in the image which are divided in accordance to a particular grid to exploit local spatial coherence. The contrast of each tile is enhanced. This is where the difference between AHE and CLAHE must be noted. In AHE, the different regions are joined together once they are enhanced in contrast without removing the induced boundaries of the tiles which adds to the noise in the background whereas in CLAHE the induced boundaries are removed by interpolation. This is where we limit the contrast and prevent the addition of noise to image especially in homogeneous areas of the image.

The main procedural steps to be noted in these process are

(i)Colour Channel Decomposition of the image,

(ii)Converting the RGB model into HSV(Hue ,saturation and Value) model,

(iii) Subjecting the image to CLAHE,

(iv) Converting the HSV channel image back to RGB composition [11].

This method can be used to enhance low contrast images. The amplification is limited by clipping of the histogram at a user specified value called clip limit. The clip limit determines the amount of noise to be removed and the level of contrast of the resultant image.

\section{IV.INTEGRATED APPROACH OF DCP AND CLAHE}

In this Section we take a look at the proposed algorithm in order to obtain a more enhanced, more haze free and a visually pleasing image of the underwater scenery. The process flow is mentioned in the figure 1 for better understanding of working of the method. Initially, it obtained Underwater Image. We will find that most images channel, and

(v) Return to the colour channel

suffer a hazy effect which is based on light conditions

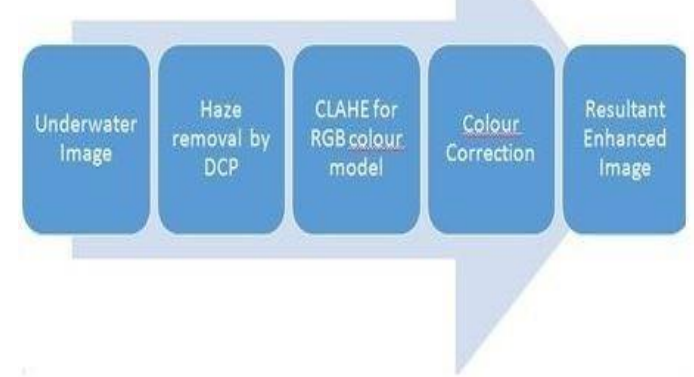

Fig. 1. Flow diagram of the Proposed Algorithm

and depth of the scenery. So it removes the haze and subject of the image to haze removing algorithm called DCP. But, on removal we found that the contrast of the image is reduced as well as darkened. In order to overcome the disadvantage of DCP, we subject the image further to CLAHE to enhance the contrast of the image where the image is divided into tiles and contrast is improved for each tile and tiles rejoined by the process of interpolation. After enhancing the contrast of the image, we further subject the image to colour correlation technique in order to get a more visually pleasing and refined image. The reason for doing this is mainly to get a better image of the object and better refinement of the image.

In Color Correlation technique we compute the Mean Square Error (MSE) and mean value in each of the RGB channels of the image say Z.

We compute the maximum and minimum value in each Channel as Follows

$$
\begin{aligned}
& Z^{c}(\max )=Z^{c}(\text { mean })+Z^{c}(\text { var }) \\
& Z^{c}(\min )=Z^{c}(\text { mean })-Z^{c}(\text { var })
\end{aligned}
$$

Where $\mathrm{C}(\mathrm{R}, \mathrm{G}, \mathrm{B})$; $\mathrm{Zc}$ (mean) is the mean value and $\mathrm{Z}^{\mathrm{c}}$ (var) is the mean square error; is the parameter to control the image dynamic.

The colour Corrected image can be computed as follows

$$
Z(c r)=\left[Z^{c}=Z^{c}(\min ) / Z^{c}(\max )-Z^{c}(\min )\right] * 255
$$

Where $\mathrm{Z}(\mathrm{cr})$ is the colour corrected image which is more refined, a better visually pleasing image, a haze free image and an image with better contrast.

\section{RESULTS AND DISCUSSION}

The evaluation of quality of the image has two types, that these are subjective and objective methods. Subjective methods are widely used for judging perceived quality of an image as it can be said that the human being is the best judge himself. The subjectively, the output of proposed method has a better quality visually than the original underwater input image.

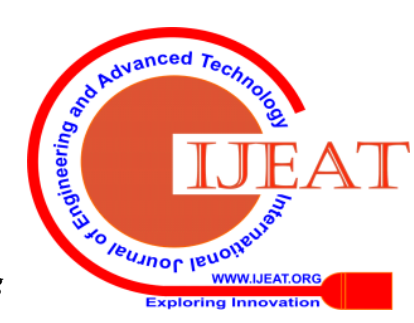




\section{An Enhancement of Underwater Images using DCP and CLAHE Algorithm}

Objectively, there are several parameters through which it can be quantitatively proved which image is better. We have considered parameters like peak signal to noise ratio, mean square error and mean absolute error.

\section{A. Mean Absolute Error}

Mean Absolute Error (MAE) is a quantity which is used to measure how close the enhanced image is to the original image. Lower the MAE, better the quality of the image. MAE can be obtained from the following formula

$$
M S E=\left(\sum_{i=1} M, N\left[S_{1}(m, n)-S_{2}(m, n)\right]^{2}\right) /(M x N)
$$

where $S_{1}$ and $S_{2}$ are denotes the original image and the enhanced image, respectively.

\section{B. Mean Absolute Error}

Mean Absolute Error (MAE) is a quantity which is used to measure how close the enhanced image is to the original image. Lower the MAE, better the quality of the image. MAE can be obtained from the following formula

$$
M A E=\left(\sum_{i=1}^{n}\left\|y_{i}-x_{i}\right\|\right) / n=\left(\left(\sum_{i=1}^{n}\left\|e_{i}\right\|\right) / n\right)
$$

the mean absolute error is an average of the absolute errors $\left\|\mathrm{e}_{\mathrm{i}}\right\|=\left\|\mathrm{y}_{i}-\mathrm{x}_{i}\right\|\left\|\mathrm{e}_{i}\right\|=\left\|\mathrm{y}_{i}-\mathrm{x}_{i}\right\|$, where $\mathrm{y}_{i}$ is the prediction and $\mathrm{x}_{i}$ is true value

\section{Peak Signal to Noise Ratio}

Peak Signal to Noise Ratio (PSNR) is the ratio between the maximum power of a signal and the power of corrupting noise. More the value of PSNR, better will be the quality of the image. PSNR is given in decibels. PSNR can be obtained from (15)

$$
\left.P S N R=-20 \log _{10}\left[2^{b}-1\right) /(\sqrt{M S E})\right]
$$

Where ' $b$ ' is the number of bits per sample Simulations for the proposed algorithm is done with the support of MATLAB Image Processing toolbox. In order to make it more visually engaging, we have designed a Graphical User Interface(GUI). That it helps us to see the image enhancement after each step in the algorithm.

\section{Experimental Analysis}

Raw Materials like Wet Sand from Beach, Pebbles of various sizes, Shells, Algae were collected to set up in an Underwater Test Tank of dimensions $120 \mathrm{~cm} \mathrm{x} 50 \mathrm{~cm} \mathrm{x}$ $20 \mathrm{~cm}$. After setting up the raw materials Sea water was poured to allow the raw materials to settle and arrive at scenery close to the one existing underwater. After pouring the water into the tank 24 hrs time was given to allow the entire setup to settle down in order for the Tyndall effect to come into play and other external factors like Atmospheric light and scattering constant to come into play. Before allowing the setup to settle down some pictures were taken using a smart phone camera of objects at various depths and after 24 hrs pictures of the same objects at the same depths were taken using the same smart phone camera. This is done for differentiating the images before and after setting in of haze due to Tyndall effect as some amount of time is required for the particles to get suspended

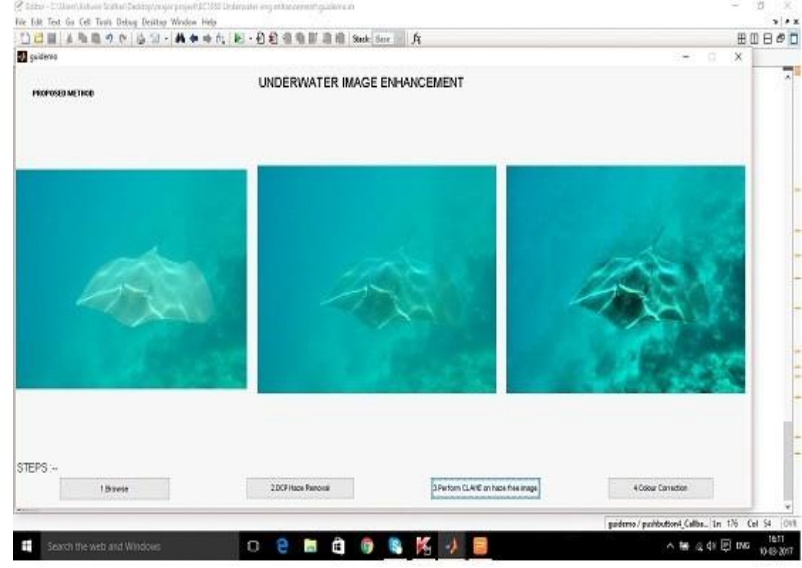

Fig 2. Enhancement of Image Using CLAHE

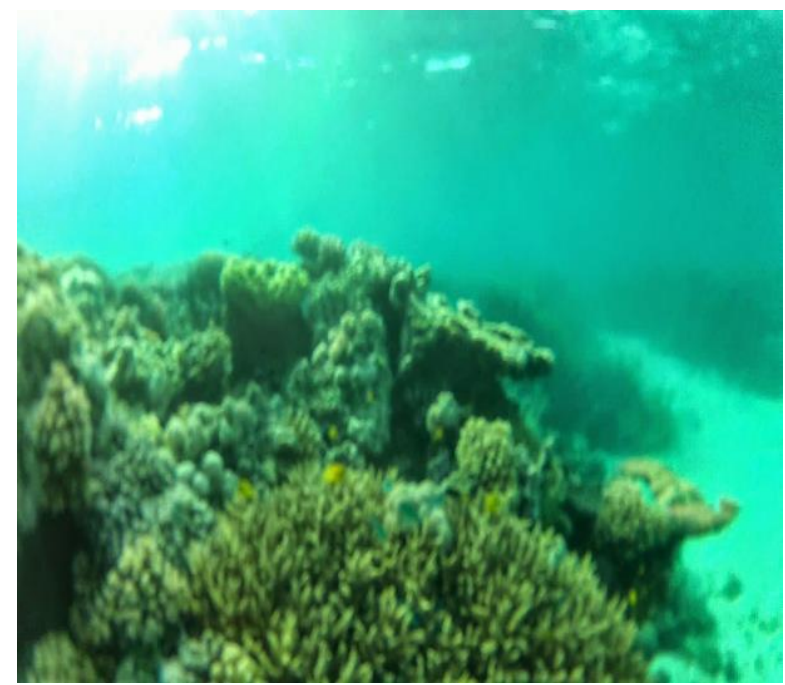

Fig. 3. Image before applying proposed algorithm

and cause non-uniform attenuation of light which is the main cause setting in of Haze. The testing part be divided into two broad cases are i)Under Clear Water Condition ii)Under Hazy Condition each of which containing various sub-cases within them. The cases may include images of sources taken from various Depths, the angle from which the images were taken, the number of sources, etc. Most of the cases showed similar results and some cases could not be tested out owing to practical difficulties coming up like field of coverage of camera, power of the camera etc. The two broad cases are further elaborated further below.

1) CASE 1: Under Clear Water Condition: As Stated earlier, in this case images of the sources were taken immediately after the sea water was poured into the tank. Three Samples have been taken up for study and parameters like PSNR, MSE and MAE are looked into for the efficiency of the algorithm. 


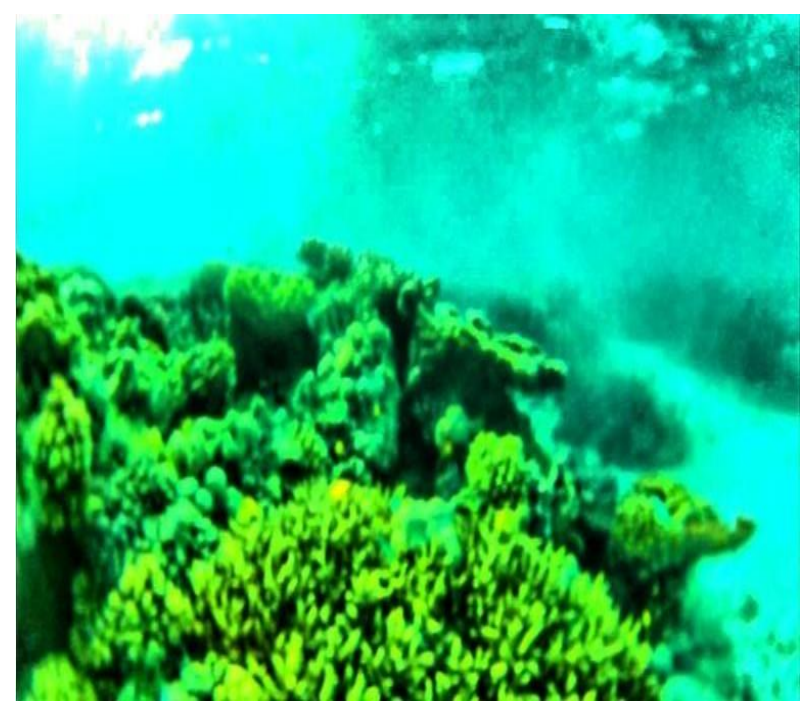

Fig. 4. Image after applying proposed algorithm

The three samples were the best cases which could provide significant results as other samples were either similar to this or had constraints with respect to the camera. Sample 1 is taken at a height of $20 \mathrm{~cm}$ from the top of the tank and the image has been taken with a normal smart phone camera as shown in fig.5. Fig.6 indicates the object after processing through the final step of the proposed approach. Here there is more than one source.

Sample 2 is taken at a height of $5 \mathrm{~cm}$ from the top of the tank and the image has been taken with a normal smart phone camera as shown in fig. 7 Fig. 8 indicates the object after processing through the final step of the proposed approach. The image is a side view image and contains a single source

Sample 3 is taken at a height of $15 \mathrm{~cm}$ from the top of the tank and the image has been taken with a normal smart phone camera as shown in fig.9. Fig.10 indicates the object after processing through the final step of the proposed approach. The image is a top view image and contains more than one source

The PSNR after following the proposed integrated approach for all the three samples was found to be higher than the values after applying DCP and CLAHE algorithms separately in all samples as is evident from the graph. When a comparative approach is carried out, it should be kept in mind that more the value of PSNR, better is the quality of the image. Therefore it can be inferred that the proposed integrated approach gives better results than the already existing single approach. The MSE after following the proposed integrated approach for all the 3 samples was found to be lower than the values after applying DCP and CLAHE algorithms separately in all samples as is evident from the graph. For the comparative study undertaken here it should be kept in mind that lower the value of MSE, better is a particular approach and going by MSE graph here it can be inferred that proposed integrated approach is better than the individual approaches of DCP and CLAHE done alone separately.

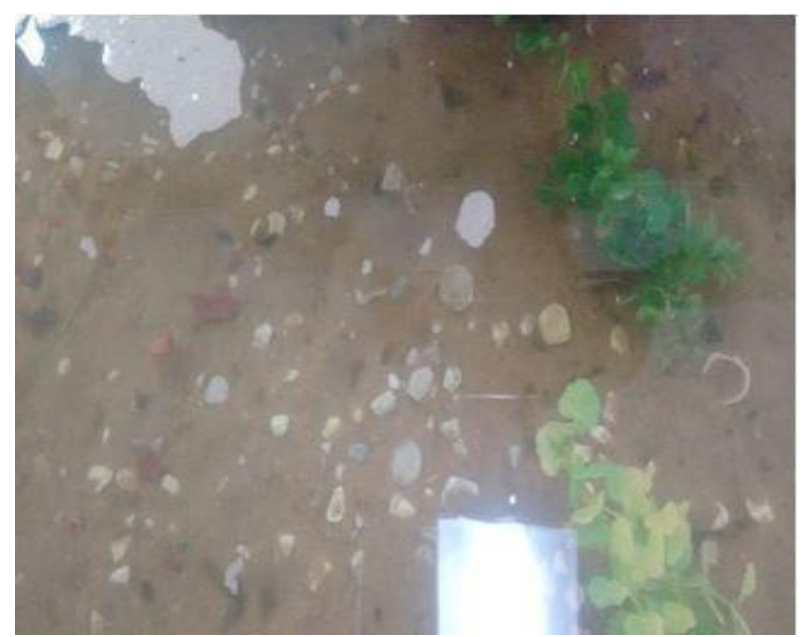

Fig. 5. Sample 1 Input Image

The MAE after following the proposed integrated approach for all the 3 samples was found to be lower than the values

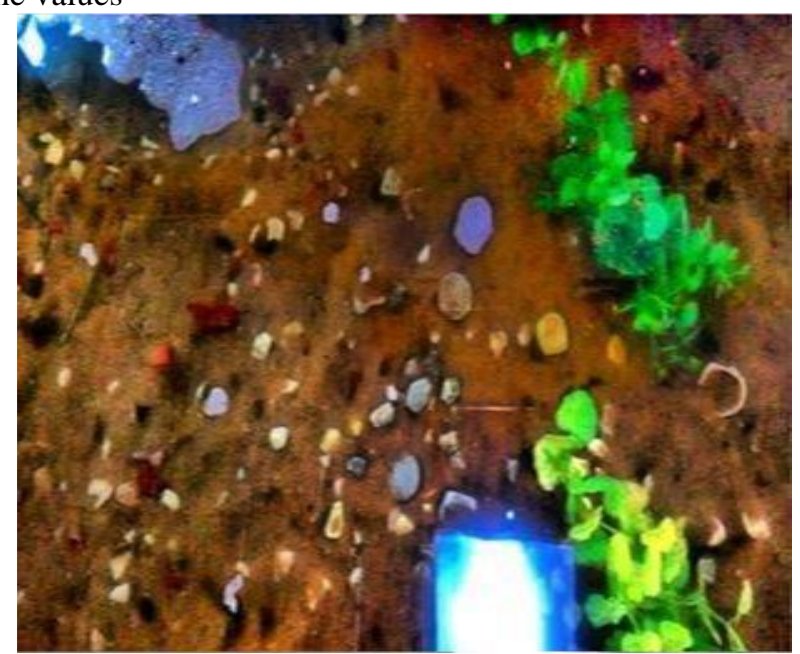

Fig. 6. Sample 1 Output Image

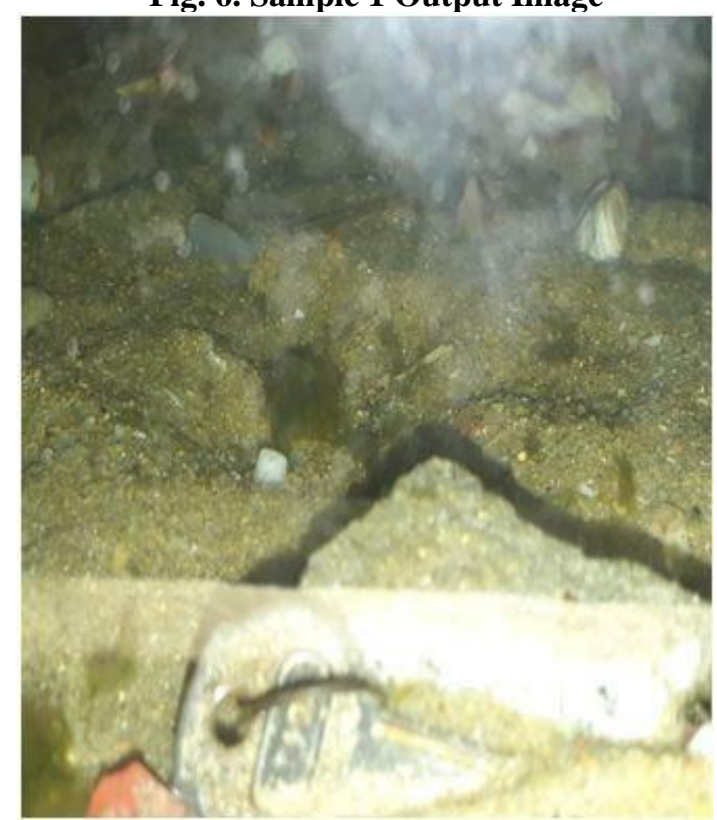

Fig. 7. Sample 2 Input Image 


\section{An Enhancement of Underwater Images using DCP and CLAHE Algorithm}

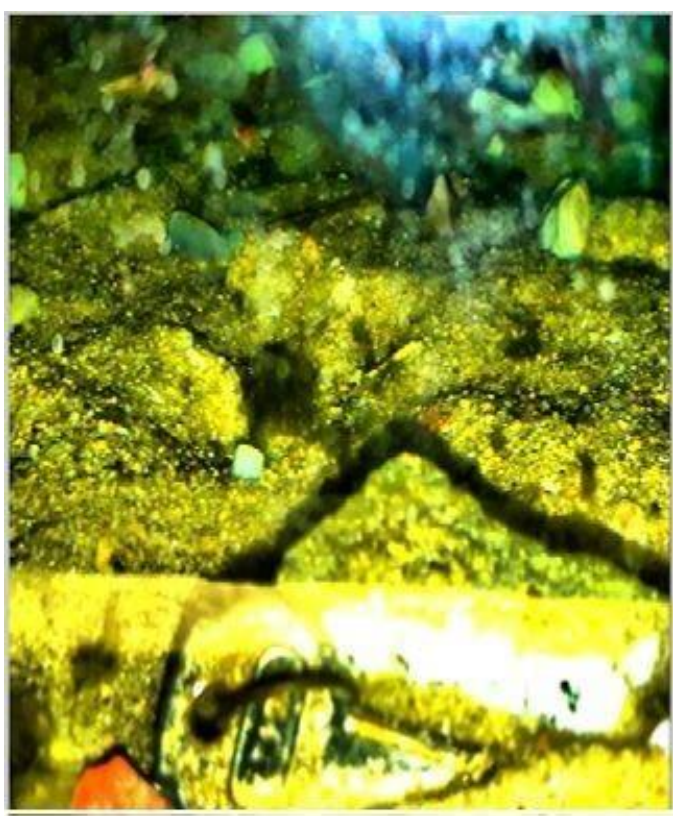

Fig. 8. Sample 2 Output Image

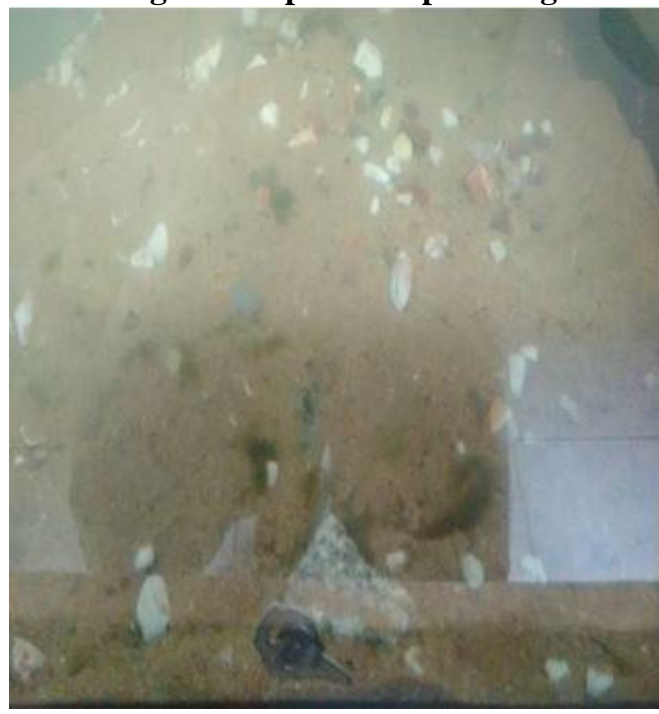

Fig. 9. Sample 3 Input Image

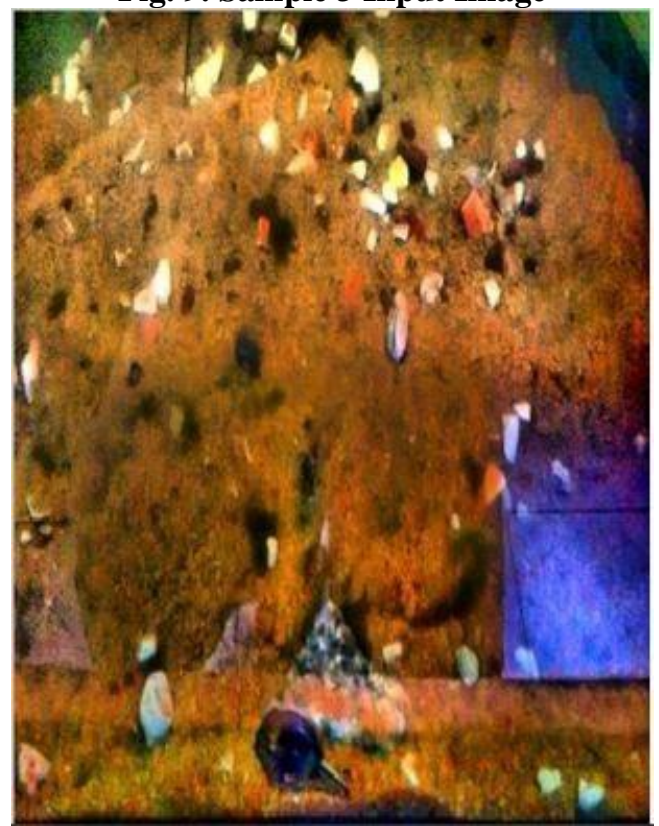

Fig. 10. Sample 3 Output Image

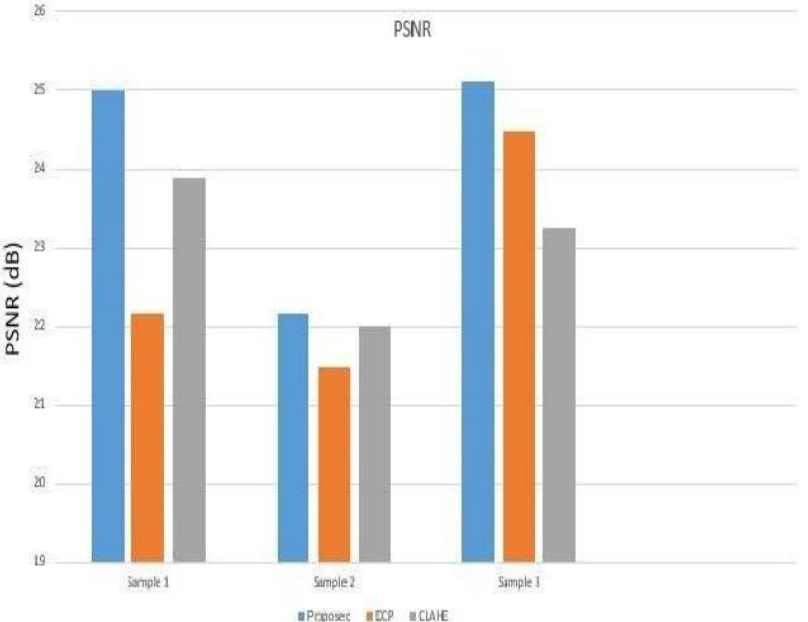

Fig. 11. Performance of PSNR

MSE

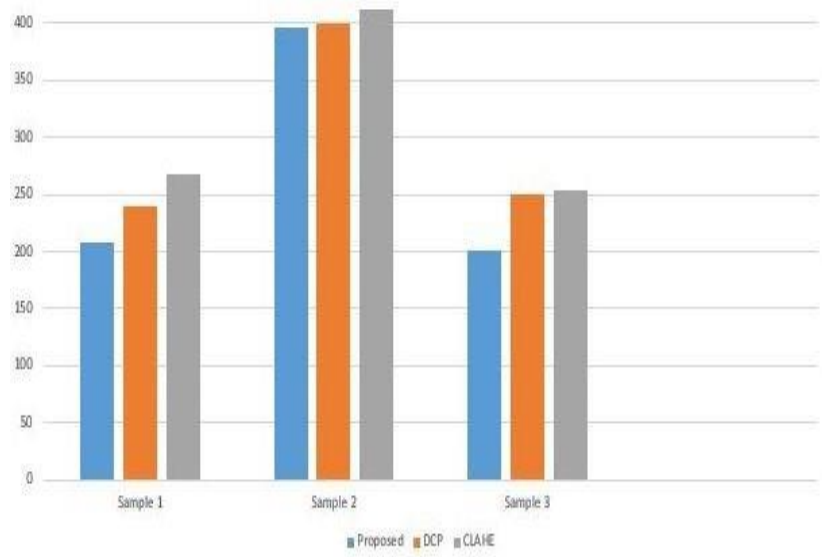

Fig. 12. Performance of MSE

after applying DCP and CLAHE algorithms separately in all samples as is evident from the graph. For the comparative study undertaken here it should be kept in mind that lower the value of MAE, better is a particular approach and going by MAE graph here it can be inferred that proposed integrated approach is better than the individual approaches of DCP and CLAHE done alone separately.

2) CASE 2: Under Hazy Condition: The images which are considered as a part of this case were taken after allowing a settling period of $24 \mathrm{hrs}$ so that Haze would settle in. Haze is mist or cloudy appearance formed due to the uneven attenuation of light as we go deeper and deeper and this would be possible only if allow the setup to settle down, so that the particles get suspended allowing Tyndall effect to creep in. Sample 1 is taken at a height of $5 \mathrm{~cm}$ from the top of the tank and the image has been taken with a normal smart phone camera as shown in fig.14. Fig.15 indicates the object after processing through the final step of the proposed 


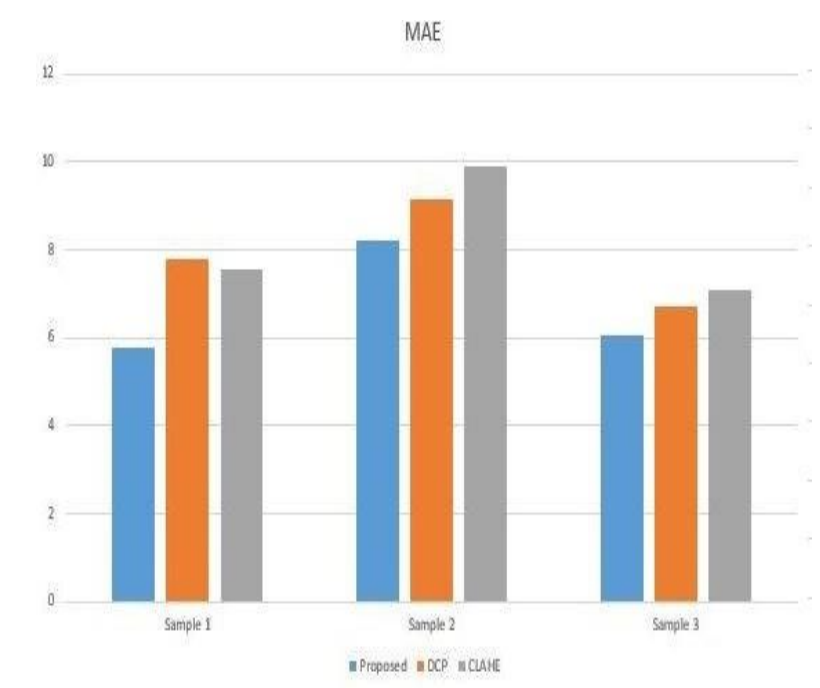

Fig. 13. Performance of MAE

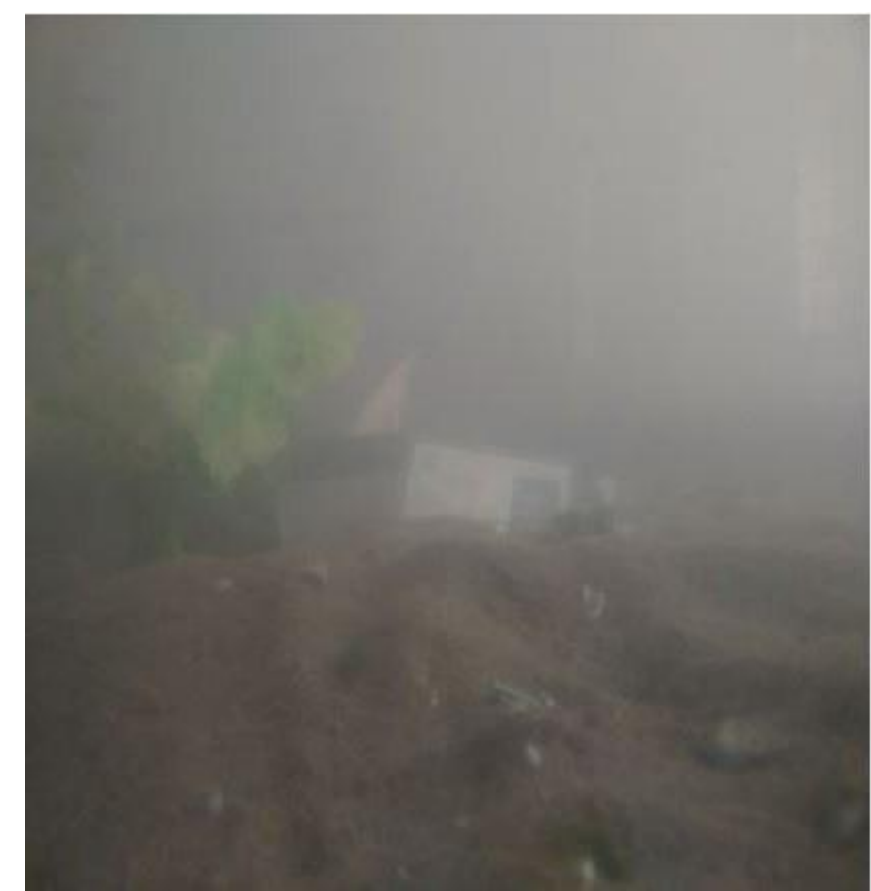

Fig. 14. Sample1 before applying Hazzy algorithm

approach. Here there is more than one source and the image is a side view image Sample 2 is taken at a height of $20 \mathrm{~cm}$ from the top of the tank and the image has been taken with a normal smart phone camera as shown in fig.16. Fig.17 indicates the object after processing through the final step of our approach. The image is a top view image Sample 3 is taken at a height of $10 \mathrm{~cm}$ from the top of the tank (side view) and the image has been taken with a normal smart phone camera as shown in fig.18. Fig.19 indicates the object after processing through the final step of our approach and is a side view image.

The PSNR after following the proposed integrated approach for all the 3 samples was found to be higher than the values after applying DCP and CLAHE algorithms separately in all samples as is evident from the graph. When a comparative approach is carried out, it should be kept in mind that more the value of PSNR, better is the quality of the image. Therefore it can be inferred that the proposed integrated approach gives better results than the already existing single approaches.

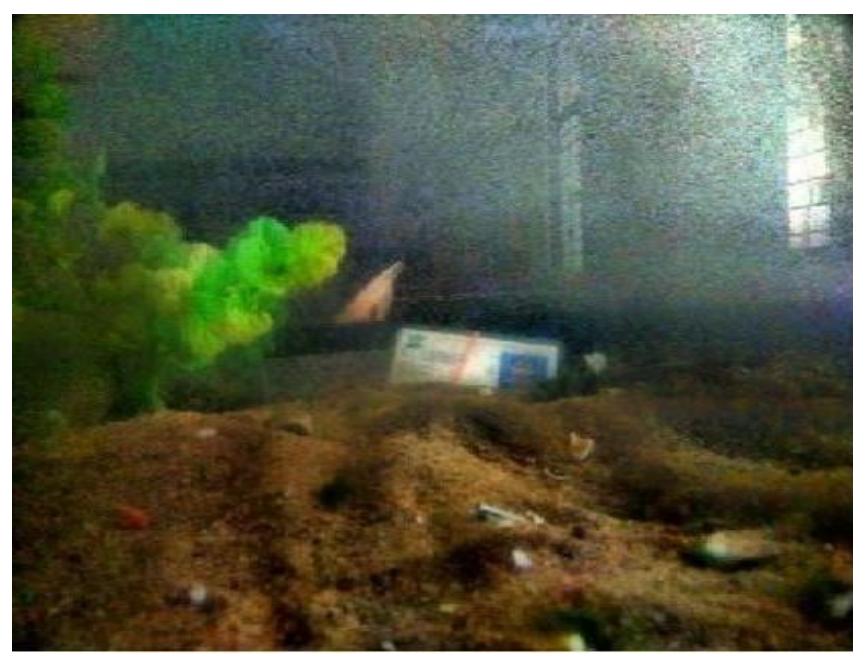

Fig. 15. Sample1 Image after applying Hazzy algorithm

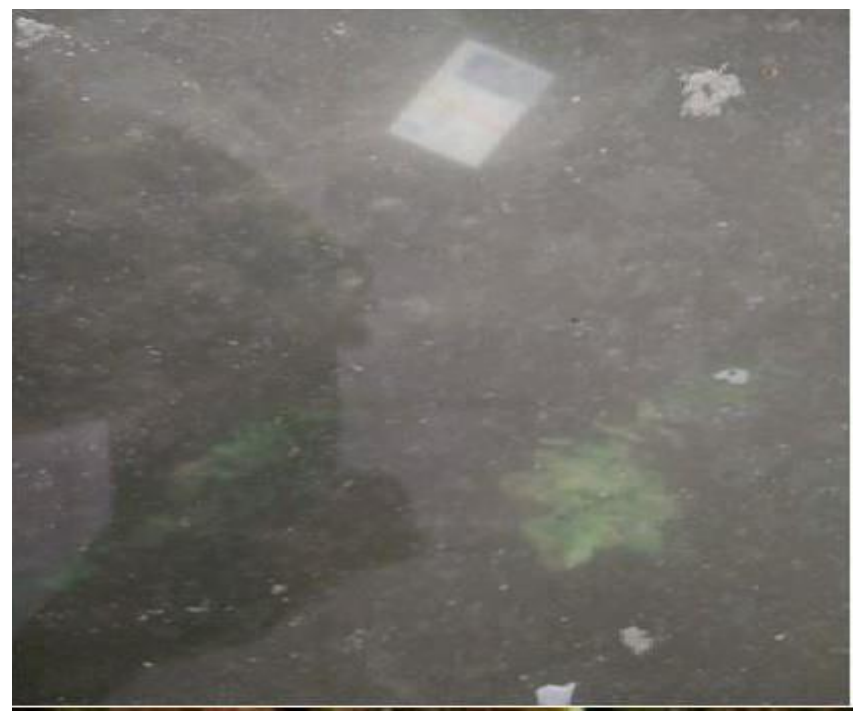

Fig. 16. Sample 2 before applying Hazzy algorithm

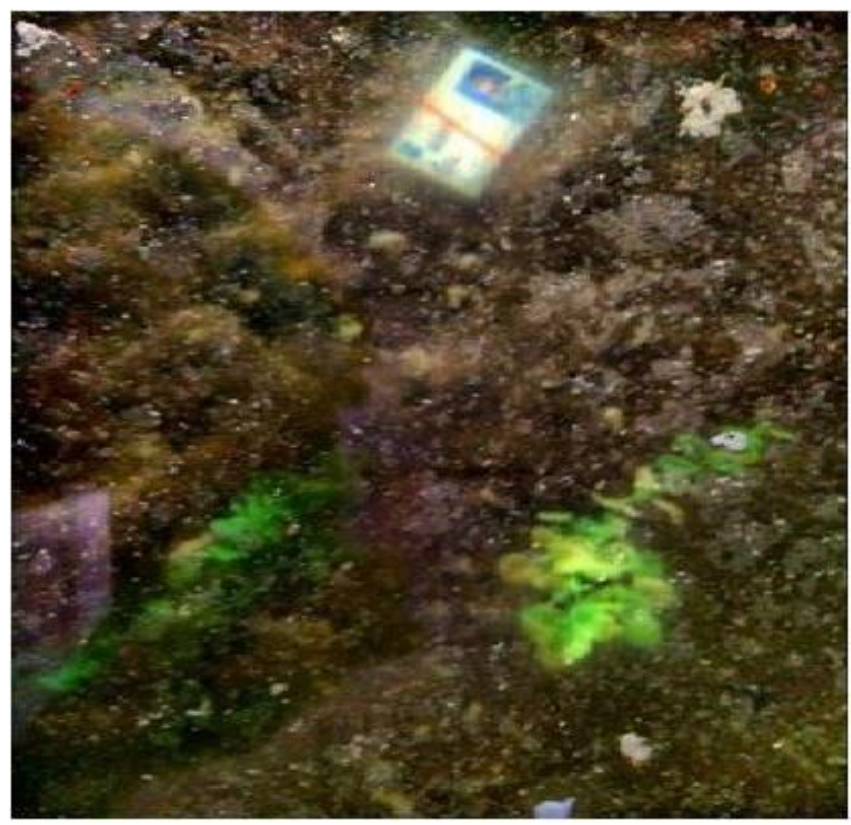

Fig. 17. Sample2 after applying Hazzy algorithm

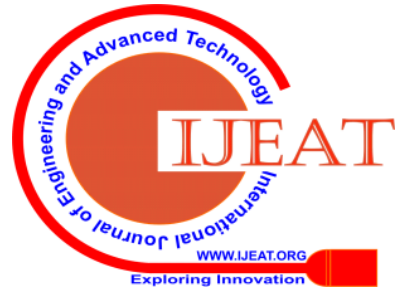




\section{An Enhancement of Underwater Images using DCP and CLAHE Algorithm}

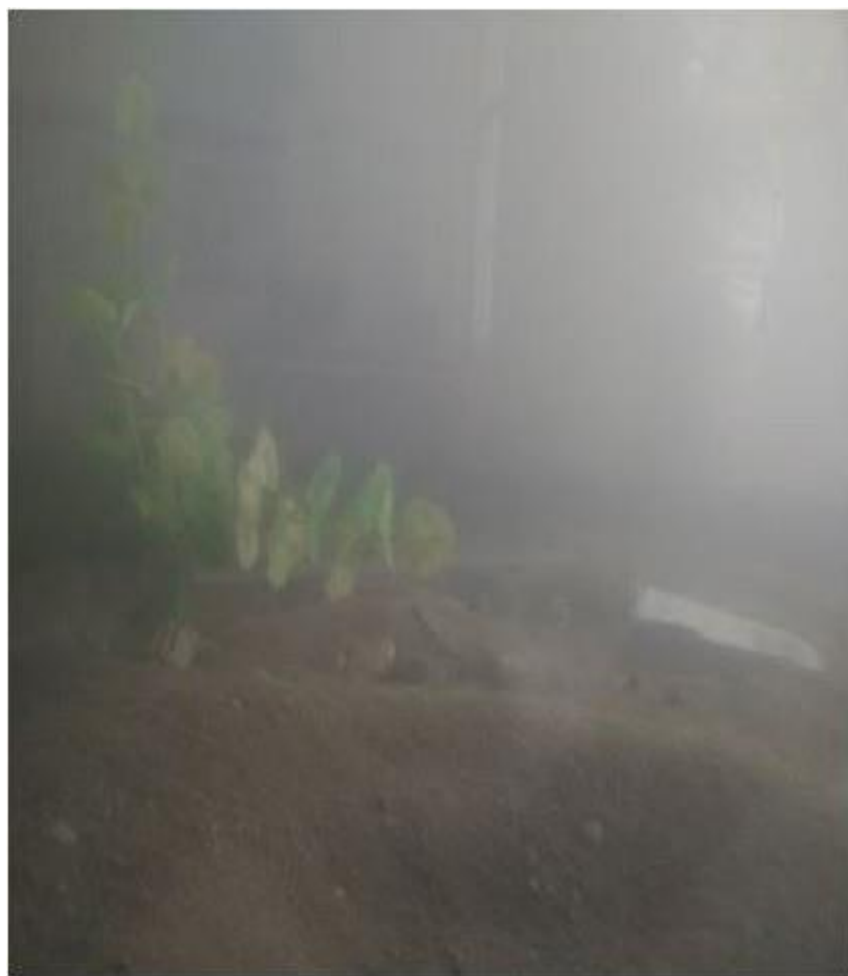

Fig 18. Sample3 before applying Hazzy algorithm

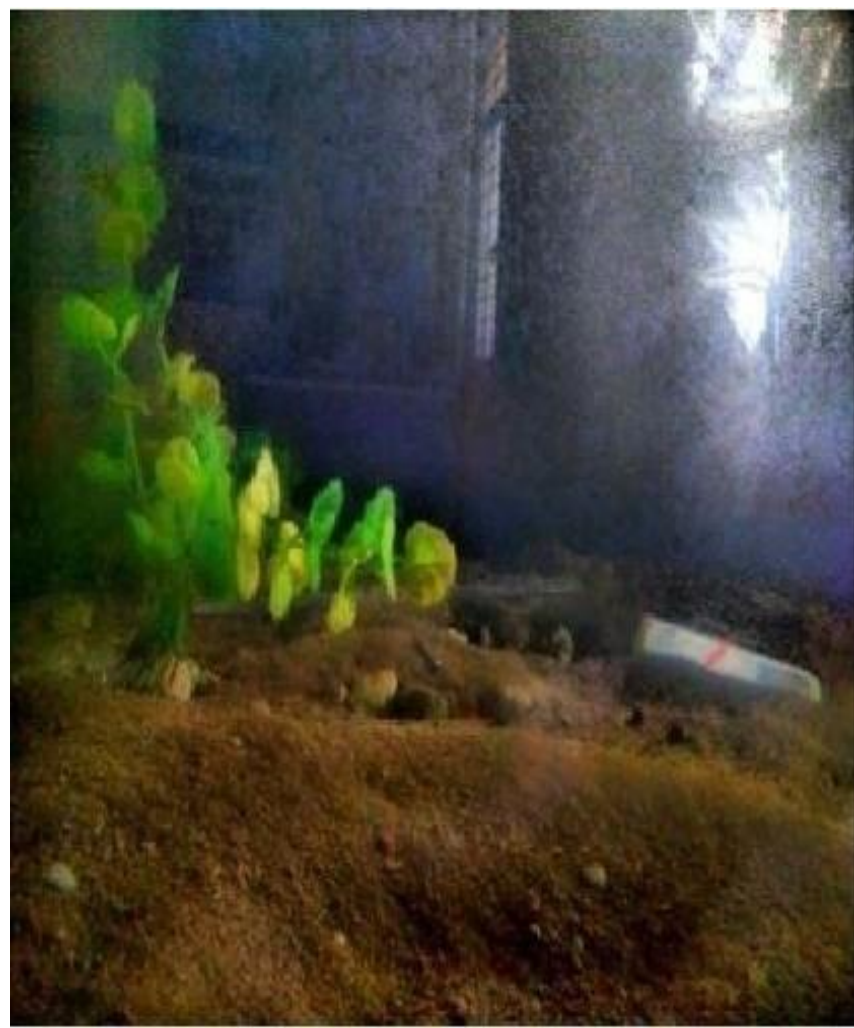

Fig. 19. Sample3 after applying Hazzy algorithm

The MSE after following the proposed integrated approach for all the 3 samples was found to be lower than the values after applying DCP and CLAHE algorithms separately in all samples as is evident from the graph. For the comparative study undertaken here it should be kept in mind that lower the value of MSE, better is a particular approach and going by MSE graph here it can be inferred that proposed integrated approach is better than the individual approaches of DCP and CLAHE done alone separately

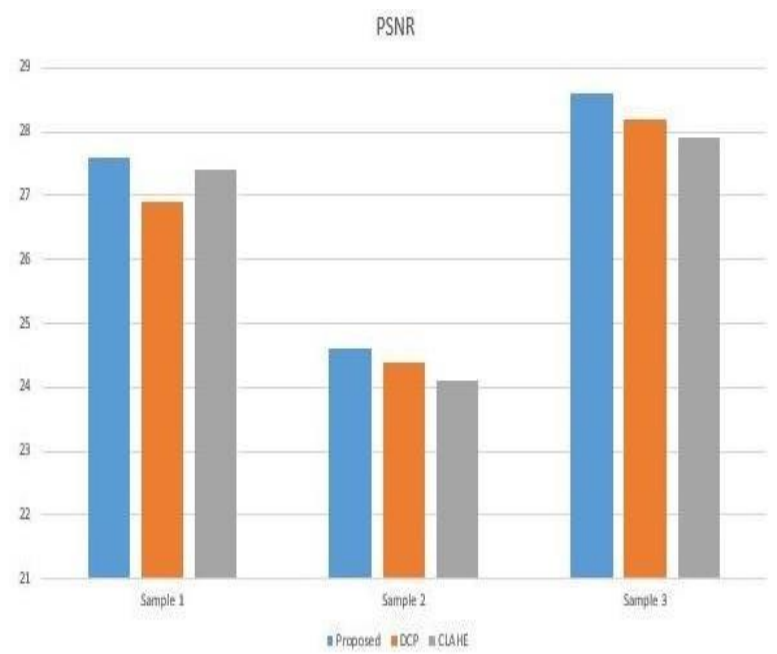

Fig. 20. Performance of PSNR

The MAE after following the proposed integrated approach for all the 3 samples was found to be lower than the values after applying DCP and CLAHE algorithms separately in all samples as is evident from the graph. For the comparative study undertaken here it should be kept in mind that lower the value of MAE, better is a particular approach and going by MAE graph here it can be inferred that proposed integrated approach is better than the individual approaches of DCP and CLAHE done alone separately.

MSE

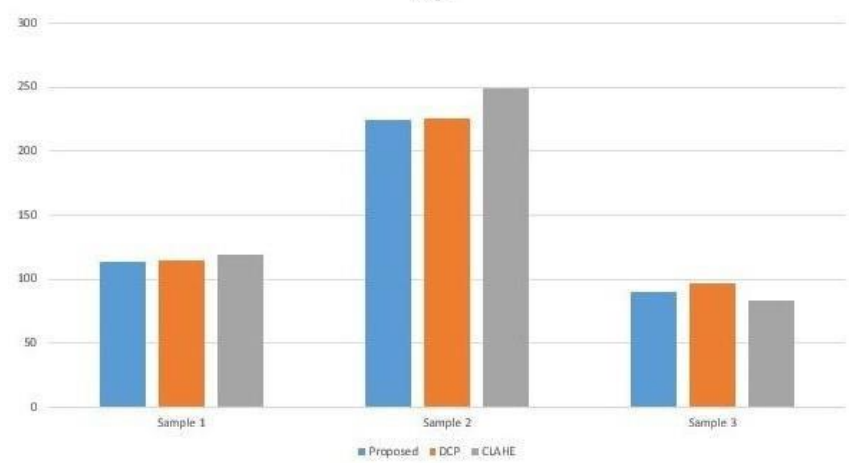

Fig. 21. Performance of MSE

MAE

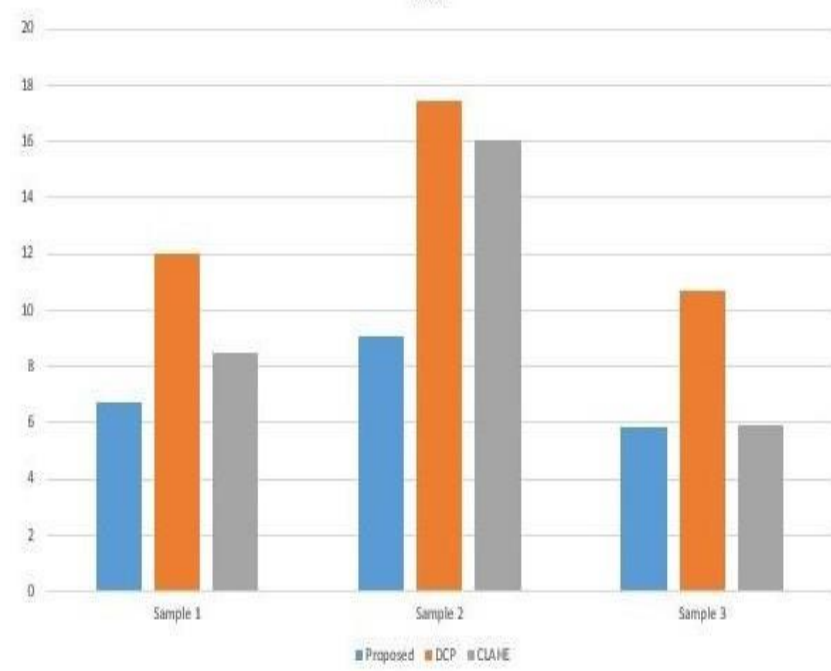

Fig. 22. Performance of MAE

Published By:

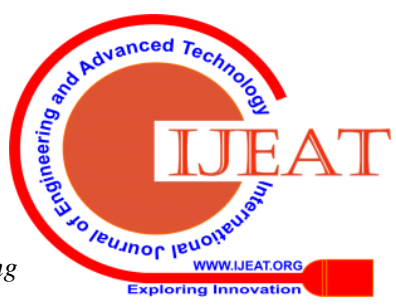




\section{CONCLUSION}

The review of the various existing methods to enhance the quality of underwater images was done.

But it have some disadvantages which came with the existing methods along with gaps in the literature survey were looked into and based on that an integrated approach combining DCP and CLAHE techniques for better quality of images is proposed. DCP method is used to remove the haze in the image. But, it was also noticed that DCP darkens the image and results in image of poor contrast. So, to enhance the contrast of the image, it was subjected to CLAHE and an image of better contrast was obtained. Finally, the image was subjected to Colour Correlation technique and got a more visually pleasing image. Objectively, with proper simulation for various images and comparison of various parameters like PSNR, MSE and MAE, it was concluded the results favored the integrated approach. Thus, it can concluded that the proposed integrated approach is better suited than the already existing methods in enhancing the quality of underwater images. This proposal part can be extended to real time research experiments in the deep seas and ocean with water proof cameras to obtain better quality and more accurate results. This algorithm can be used in applications like artificial intelligence, computer vision and military applications such as in submarines for taking pictures of the debris of a plane crash or ship wreck at the ocean bed. This algorithm can also be supported and extended for identifying city submerged underwater which has been there for long years.

\section{REFERENCES}

1. C. Ancuti, C. O. Ancuti, T. Haber, and P. Bekaert, "Enhancing underwater images and videos by fusion," in Computer Vision and Pattern Recognition (CVPR), 2012 IEEE Conference on, pp. 81-88, IEEE, 2012.

2. R. Fattal, "Single image dehazing," ACM transactions on graphics (TOG), vol. 27, no. 3, p. 72, 2008.

3. M. S. Hitam, E. A. Awalludin, W. N. J. H. W. Yussof, and Z. Bachok, "Mixture contrast limited adaptive histogram equalization for underwater image enhancement," in Computer Applications IEEE, 2013.

4. C. O. Ancuti, C. Ancuti, C. De Vleeschouwer, and P. Bekaert, "Color balance and fusion for underwater image enhancement," IEEE Transac- tions on Image Processing, vol. 27, no. 1, pp. 379-393, 2018.

5. K. He, J. Sun, and X. Tang, "Single image haze removal using dark channel prior," IEEE transactions on pattern analysis and machine intelligence, vol. 33, no. 12, pp. 2341-2353, 2011.

6. C.-Y. Li, J.-C. Guo, R.-M. Cong, Y.-W. Pang, and B. Wang, "Underwater image enhancement by dehazing with minimum information loss and histogram distribution prior," IEEE Transactions on Image Processing, vol. 25, no. 12, pp. 5664-5677, 2016.

7. R. Fries and J. Modestino, "Image enhancement by stochastic homomorphic filtering," IEEE Transactions on Acoustics, Speech, and Signal Processing, vol. 27, no. 6, pp. 625-637, 1979.

8. K. Kim, S. Kim, and K.-S. Kim, "Effective image enhancement techniques for fog-affected indoor and outdoor images," IET Image Processing, vol. 12, no. 4, pp. 465-471, 2017.

9. A. K. Tripathi and S. Mukhopadhyay, "Single image fog removal using bilateral filter," in Signal Processing, Computing and Control (ISPCC), 2012 IEEE International Conference on, pp. 1-6, IEEE, 2012.

10. T. H. Kil, S. H. Lee, and N. I. Cho, "A dehazing algorithm using dark channel prior and contrast enhancement," in Acoustics, Speech and Signal Processing (ICASSP), 2013 IEEE International Conference on, pp. 2484-2487, IEEE, 2013.

11. S.L.Wong, Y. P. Yu, N. A. J. Ho, and R. Paramesran, " Comparative Technology (ICCAT), 2013 International Conference on, pp. 1-5,

analysis of underwater image enhancement methods in different color spaces ," 2014 International Symposium on Intelligent Signal Processing and Communication Systems (ISPACS), pp. 034-038, 2014

\section{AUTHORS FROFILE}

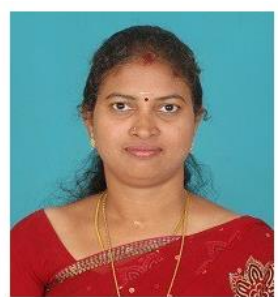

Ms.V.Malathi, received her bachelors Degree in 2000 and Master degree in 2004 from University of Madras, Tamilnadu, India and M.Phil from Periyar University, Tamilnadu, India in 2009. She currently pursuing Ph.D at Periyar University. She has 10 years of teaching experience. Currently, she is work- ing as a Assistant Professor, SRM Arts Science college, Chennai, Tamilnadu, India. Her research interests are in Image processing, Wireless Sensor Networks and Network Security. She also published her research works in various international journals and presented in many national and international conferences.

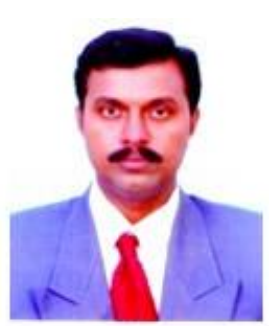

Dr. A.Manikandan, received bachelors Degree in 1997, Master degree in 1999 from Bharathidasan University, Tamilnadu, India and M.Phil from M.S University, Tamilnadu, India in 2003. He completed M.Tech in 2011 from PRIST University, Tamilnadu, India. He did his Ph.D in 2017 at Dravidian University, Andra Pradesh, India. He has 20 years of teaching experience. Currently, he is working as a Principal Assistant Professor, Muthayammal Memo- rial College of Arts Science, Rasipuram, Tamilnadu, India. His research interests are in Wireless Ad Hoc Networks, Wireless Sensor Networks, Network Security, Image Processing and Data Mining. He is a life member of Indian Society for Technical Education. He published 22 national International journals and also published Programming in C, Embedded Systems Web Technology (Bharathi Publishers) and Computer Programming (Global Publishers). He is being guided 4 research scholars. He is a board member of various journals and also member in professional bodies of ISTE, TNSRO, IARA TERA.

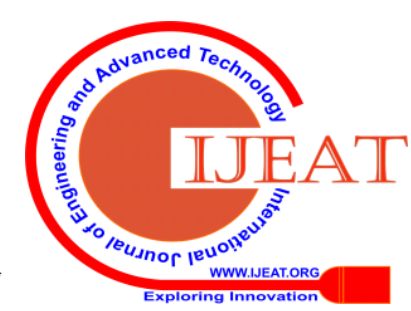

